\title{
MISINTERPRETATION OF HISTOMORPHOLOGICAL CRITERIA IN APOPTOSIS AND SIGNIFICANCE OF IMMUNOHISTOCHEMISTRY
}

\author{
Marian Adamkov \\ Department of Histology and Embryology, Jessenius Faculty of Medicine, Comenius \\ University, Martin, Slovakia
}

\section{Dear Editor,}

apoptosis or programmed cell death is one of the key players in orchestrating normal development of embryonic and fetal tissues and organs. Generally, apoptosis is responsible for negative regulation of cell proliferation activities. Briefly, during developmental processes, apoptosis is involved in elimination of useless and redundant cells, thus controlling proper cell number and size of organs (Galluzzi et al., 2018; Voss and Strasser, 2020).

Since apoptosis is unique mechanism to remove unwanted cells, I have read with appropriate solemnity original article "Transformation of the human prostate gland in intermediate and late fetal periods of development" by I. Piatsko and A. Usovich published in Rev Arg de Anat Clin (2020; 12(3): 131-136).

Above mentioned article represents histomorphological study and therefore let me draw attention on Figure 1 and Figure 2. Firstly, I would like to stress widely accepted basic histomorphological features of cells committed to apoptotic cell death. Apoptosis is well known by its stereotyped morphology. In early phase, apoptotic cells are rounded and shrunk. The intracellular water is reduced through plasma membrane channels. Cells become smaller in size, the organelles are tightly packed and cytoplasm is dense. Smaller cells are situated within unstained "halo". Therefore, these cellular elements are easily diagnosed by light microscopy. Prominent feature of apoptosis is widespread chromatin condensation with its marginalization. Later, chromatin is fragmented and affected cells form numerous apoptotic bodies, which are quickly phagocytosed by macrophages and neighbouring cells (Elmore, 2007; Galluzzi et al., 2015; D’Arcy, 2019; Voss and Strasser, 2020).

In Figure 1, arrow 2 shows "apoptotic cells". The arrow is pointing to the dark nucleus located basally. Considering basic histomorphological criteria, there is strong hesitation to be positively "apoptotic cell". We can recognize numerous nuclei in basal location of shown ducts with very similar morphology. From morphological point of view, it is probably nucleus of basal cell. In such cases, it is recommended to use immunohistochemistry for exact diagnosis of apoptotic cells, e.g. TUNEL, M30 Cyto Death, Annexin V, caspase 3, etc. (Elmore, 2007; Adamkov, 2020). In Figure 2, A indicates "conglomerates of apoptotic cells in the prostatic ductal lumens". To this statement, I would like to underline that apoptotic cells may form conglomerates very rarely. Usually, single cells undergo apoptotic machinery, it begins within epithelium or other type of tissue. Furthermore, cells forming conglomerate in Figure 2 do not possess apoptotic features at all (see above). Already at the first glance, they are very large including nuclei with wide rim of cytoplasm.

In summary, I would like to point out significance of precise interpretation of basic histomorphological criteria for apoptotic cell death. If cellular histomorphology raises doubts, immunohistochemical approaches may offer numerous ways to disclose cells in various stages of apoptotic cascade.

\footnotetext{
* Correspondence to: Prof. Marian Adamkov.
} 


\section{References}

Adamkov M. 2020. Apoptosis and anti-apoptotic protein survivin as promising tumor biomarker. 1st ed.Germany: Rank. $156 \mathrm{p}$.

D'Arcy MS. 2019. Cell death: a review of the major forms of apoptosis, necrosis and autophagy. CellBiollnt. 43: 582-92.

Elmore S. 2007. Apoptosis: A review of programmed cell death. Toxicologic Pathology. 35: 495-516.

Galluzzi L, Bravo-San Pedro JM, Vitale I, Aaronson SA, Abrams JM, Adam D, Alnemri $E S$, Altucci $L$, Andrews $D$, AnnicchiaricoPetruzzelli M, Baehrecke EH, Bazan NG, Bertrand MJ, Bianchi K, Blagosklonny MV, Blomgren K, Borner C, Bredesen DE, Brenner C, Campanella M, Candi E, Cecconi F, Chan FK, Chandel NS, Cheng EH, Chipuk JE, Cidlowski JA, Ciechanover A, Dawson TM, Dawson VL, De Laurenzi $V$, De Maria $R$, Debatin K-M, Di Daniele N, M Dixit $V$, Dynlacht BD, El-Deiry WS, Fimia GM, Flavell RA, Fulda S, Garrido C, Gougeon M-L, Green $D R$, Gronemeyer H, Hajnoczky G, Hardwick $J M$, Hengartner $M O$, Ichijo $H$, Joseph B, Jost PJ, Kaufmann T, Kepp O, Klionsky DJ, Knight RA, Kumar S, Lemasters JJ, Levine B, Linkermann A, Lipton SA, Lockshin RA, LópezOtín C, Lugli E, Madeo F, Malorni W, Marine JC, Martin SJ, Martinou J-C, Medema JP, Meier $P$, Melino $S$, Mizushima $N$, Moll $U$, MuñozPinedo C, Nuñez G, Oberst A, Panaretakis $T$, Penninger JM, Peter ME, Piacentini M, Pinton $P$, Prehn JH, Puthalakath $H$, Rabinovich $G A$, Ravichandran KS, Rizzuto $R$, Rodrigues CM, Rubinsztein DC, Rudel T, Shi Y, Simon HU, Stockwell BR, Szabadkai G, Tait SW, Tang $H L$, Tavernarakis $N$, Tsujimoto $Y$, Vanden Berghe $T$, Vandenabeele $P$, Villunger $A$, Wagner EF, Walczak H, White E, Wood WG, Yuan J, Zakeri Z, Zhivotovsky B, Melino G, Kroemer G. 2015. Essential versus accessory aspects of cell death: recommendations of the NCCD 2015. Cell Death and Differentiation. 22: 58-73.

Galluzzi L, Vitale I, Aaronson SA, Abrams JM, Adam D, Agostinis P, Alnemri ES, Altucci L, Amelio I, Andrews DW, AnnicchiaricoPetruzzelli M, Antonov AV, Arama E, Eric $H$, Baehrecke EH, Barlev NA, Bazan NG, Bernassola F, Bertrand MJM, Bianchi K,
Blagosklonny MV, Blomgren K, Borner C, Boya $P$, Brenner $C$, Campanella $M$, Candi $E$, Carmona-Gutierrez D, Cecconi F, Chan FK-M, Chandel NS, Cheng EH, Chipuk JE, Cidlowski $J A$, Ciechanover A, Cohen GM, Conrad M, Cubillos-Ruiz JR, Czabotar PE, D'Angiolella $V$, Dawson TM, Dawson VL, De Laurenzi V, De Maria $R$, Debatin K-M, DeBerardinis RJ, Deshmukh M, Di Daniele N, Di Virgilio F, Dixit VM, Dixon SJ, Duckett CS, Dynlacht BD, ElDeiry WS, Elrod JW, Fimia GM, Fulda S, GarcíaSáez AJ, Garg AD, Garrido C, Gavathiotis E, Golstein P, Gottlieb E, Green DR, Greene LA, Gronemeyer $H$, Gross A, Hajnoczky $G$, Hardwick JM, Harris IS, Hengartner MO, Hetz $C$, Ichijo $H$, Jäättelä $M$, Joseph $B$, Jost $P J$, Juin $P P$, Kaiser WJ, Karin M, Kaufmann T, Kepp $O$, Kimchi $A$, Kitsis RN, Klionsky DJ, Knight RA, Kumar S, Lee SW, Lemasters JJ, Levine B, Linkermann A, Lipton SA, Lockshin RA, LópezOtín C, Lowe SW, Luedde T, Lugli E, MacFarlane M, Madeo F, Malewicz M, Malorni W, Manic G, Marine J-C, Martin SJ, Martinou J$C$, Medema JP, Mehlen P, Meier P, Melino S, Miao EA, Molkentin JD, Moll UM, Muñoz-Pinedo $C$, Nagata $S$, Nuñez $G$, Oberst $A$, Oren $M$, Overholtzer $M$, Pagano $M$, Panaretakis $T$, Pasparakis M, Penninger JM, Pereira DM, Pervaiz S, Peter ME, Piacentini M, Pinton $P$, Prehn JHM, Puthalakath $H$, Rabinovich GA, Rehm $M$, Rizzuto $R$, Rodrigues CMP, Rubinsztein $D C$, Rudel T, Ryan KM, Sayan E, Scorrano L, Shao F, Shi Y, Silke J, Simon HU, Sistigu A, Stockwell BR, Gyorgy Szabadkai $S A$, Tait SWG, Tang D, Tavernarakis $N$, Thorburn A, Tsujimoto $Y$, Turk B, Vanden Berghe $T$, Vandenabeele $P$, Vander Heiden $M G$, Villunger $A$, Virgin HW, Vousden $K H$, Vucic $D$, Wagner $E F$, Walczak $H$, Wallach $D$, Wang $Y$, Wells JA, Wood W, Yuan J, Zakeri Z, Zhivotovsky B, Zitvogel L, Melino G, Kroemer G. 2018. Molecular mechanisms of cell death: recommendations of the Nomenclature Committee on Cell Death 2018. Cell Death \& Differentiation. 25: 486-541. doi:10.1038/ s41418-017-0012-4

Voss AK, Strasser A. 2020. The essentials of developmental apoptosis [version 1; peer review: 3approved]. F1000Research. 9(148). https://doi.org/10.12688/f1000research.21571.1 